\title{
DISCUSSION
}

\section{Greenfield tunnelling in sands: the effects of soil density and relative depth}

\author{
A. FRANZA*, A. M. MARSHALL†, B. ZHOU†, N. SHIRLAW§ and S. BOONE\|
}

\begin{abstract}
Contribution by N. Shirlaw and S. Boone
The discussion contributors thank the authors for their paper summarising the results of 15 centrifuge tests on model tunnels in sand. Their paper represents a helpful contribution toward better quantifying the response of granular soils to tunnelling. It is the discussers' opinion that much more use could be made of centrifuge testing on model tunnels in sand to solve practical tunnelling problems. While there are numerous published centrifuge model tests in sand, including the authors', the data are typically from studies that are very limited both in their nature and in terms of possible practical application. For useful application, more tests are needed that represent real heading geometry and in saturated, rather
\end{abstract} than dry, sand.

A tunnel driven in sand typically requires a support pressure, as provided by a pressurised tunnel-boring machine (PTBM). An important aspect of geotechnical analysis for use of PTBMs is to define target values for the pressure at the face and for the tail void grouting consistent with subsurface conditions (Shirlaw \& Boone, 2009) and compliance with maximum specified values for surface settlement or surface volume loss, $V_{1, \mathrm{~s}}$. Maximum values for settlement or surface volume loss are typically set by the designer of the tunnelling project, while the pressures at the PTBM are set by the contractor or their engineer.

In their paper, the authors categorise volume loss at the tunnel level, $V_{1, t}$, of $1 \%$ as 'low' and $5 \%$ as 'high'. In practice, designers commonly require that a maximum surface volume loss of $1 \%$ is consistently achieved over PTBM tunnels in sand. The discussers have seen recent examples where a maximum value for surface volume loss of $0 \cdot 5 \%$ has been specified for tunnels in granular soil. Reviewing the authors' Figure 9 in the range from 0 to $1 \%$ tunnel volume loss, the loose and medium-dense sands $\left(I_{\mathrm{d}} \quad 0.3\right.$ and 0.5$)$ are contractive, such that the surface volume loss is significantly greater than the tunnel volume loss at all values of $C / D$. In dense sand $\left(I_{\mathrm{d}}=0.9\right)$ the ground deforms at close to constant volume, with the surface and tunnel volume loss being similar. A contractor's engineer with a target of $0.5 \%$ maximum surface volume loss will therefore have to target values for volume loss at tunnel level that are significantly less than the desired maximum value for the surface volume loss in loose and medium-dense sand. Even in

* ETSI Caminos, Universidad Politécnica de Madrid, Madrid, Spain (Orcid:0000-0002-8510-0355).

$\dagger$ Department of Civil Engineering, University of Nottingham, Nottingham, UK (Orcid:0000-0003-1583-1619).

* Formerly, Faculty of Engineering, University of Nottingham; now Shanghai Civil Engineering Co., Ltd of China Railway Group Ltd, Shanghai, P. R. China.

$\S$ Golder Associates (HK) Limited, Wan Chai, Hong Kong, P. R. China.

|| Golder Associates Ltd., London, Ontario, Canada. dense sand, the target value for volume loss at tunnel level cannot exceed the maximum value for surface volume loss.

In the discussers' opinion, the very low values of maximum surface volume loss that are now commonly specified on tunnelling projects reflect a desire to fully use the capabilities of PTBMs to control ground movement. Controlling the ground movement at source becomes the primary, and often only, means of protecting buildings, utilities and other structures from damage during PTBM tunnelling. This approach transfers much of the settlement control problem from the project designer to the contractor's engineer. The contractor's engineer has the task of defining the necessary operating pressures for tunnelling in order to consistently perform below the specified maximum values for surface volume loss. Potentially, data from testing of model tunnels in geotechnical centrifuges could provide a useful basis for making this assessment. However, data currently available are not sufficient for this task.

Frequently, when assessing potential near-surface implications of tunnelling through soft ground in urban environments, engineers apply well-known empirical methods that relate the unit volume of the ground surface settlement trough to the unit volume of the tunnel, expressed as 'volume loss', and characterise the shape of the settlement trough as an inverted standard normal probability distribution curve (e.g. Peck, 1969; New \& O'Reilly, 1981; and others). While useful for elementary studies, these methods do not adequately address the roots of surface volume loss that occur at the tunnel level, as related to closure of the ground around tunnelling machines and linings, the effects of the attitude of tunnelling machines in very soft soils or when negotiating curves and face pressure control. The pressures applied at the face, along the shield and at the tail void are critical in the control of tunnel and surface volume loss. Until fundamental research and field data quantitatively examine the contributions of each ground loss source, field control will be dominated by subjective opinion, 'rules of thumb', different methodologies and trial-and-error field adjustments.

Although graphs relating internal support pressure to tunnel volume loss are provided in the paper, these data cannot be readily applied in the assessment of support pressures for PTBM driven tunnels, as the true field problem is three-dimensional (3D) and progressive rather than the stationary plane-strain conditions of the authors' tests. The authors show how a two-dimensional (2D) arch develops over the model tunnel, at least for the higher values of soil relative density, similar to work by Iglesia et al. (2013) and others. In practice, Wan et al. (2019) illustrate the 3D propagation and sequence of soil arches and displacement modes that develop over tunnels in London Clay at various stages of tunnel advancement at the face, around the shield and around the lining, with longitudinal as well as transverse arches. While the response of sand will be different to that of clay, the obvious 3D influences of the tunnelling processes are similar. In practice, the 3D relationships between support pressures, forward tunnelling rate and the resultant surface 
volume losses will therefore be significantly different from those measured in the stationary $2 \mathrm{D}$ tests presented by the authors and stationary face loss tests presented by others. This comment is not intended to be critical of the study carried out by the authors, but to use that study as a starting point to discuss the limitations of the current 'state of the art' as applied to the practical issues involved in setting target operating pressures for PTBM tunnelling and how centrifuge modelling of tunnel processes and pressure might assist in this process.

For tunnels in intact clay, detailed results are available from centrifuge tests on model tunnels (Atkinson \& Mair (1981), Kimura \& Mair (1981), Mair et al. (1981) among many others). The results of these tests can be used as a starting point to assess the relationship between the support pressure and the surface volume loss and to carry out analysis both at ultimate limit state and serviceability limit state (ULS and SLS, respectively). The value of centrifuge test results for model tunnels in intact clay is recognised in the guidelines for PTBM pressure assessment in Hong Kong (Golder Associates (HK) Ltd, 2009; CEDD, 2014). However, the situation is different for tunnels in sand, especially when saturated, as the test data that have been published are limited, and often not directly applicable to the problem of ground movements around and over a PTBM.

For tunnels in sand, there are a variety of methods to establish the support pressure at failure, as discussed in DAUB (2016). However, establishing the relationship between the support pressure and volume loss at the tunnel or surface levels is problematic.

As discussed in the paper by Shirlaw (2012), the development of modern PTBMs, particularly for urban tunnelling, has led to the specification of ever lower limits on the allowable values for surface volume loss, while allowing the construction of very large and/or shallow tunnels in extremely challenging ground conditions. It is implicitly assumed that low values of volume loss can be reasonably predicted, planned and routinely achieved. However, too frequently, the tunnel performance expectations in such cases have been based on limited empirical data and application of older analysis methodologies, which are then extrapolated to new higher risk and more tightly specified performance conditions, without proper justification. The development of a robust framework for assessing the relationship between support pressure and surface volume loss is therefore necessary. However, unlike the situation for intact clay, there is a limited basis for this assessment for tunnels in sand.

Currently, there are three common methods for assessing the pressure needed to control surface volume loss over PTBM tunnels in sand

(a) a semi-quantitative factor of safety approach, in which a single calculation for the required factor of safety against failure provides the pressure that is deemed to control both the surface volume loss and the risk of failure in which the surface volume loss estimate is based largely on empirical case history data

(b) a simplistic SLS analysis that uses an analogy to the design load for rigid tunnel linings to assess the support pressure required at small values for ground movement (Golder Associates (HK) Ltd., 2009; CEDD, 2014)

(c) numerical methods, such as finite-element or finite-difference analysis (2D or 3D).

Methods $(a)$ and $(b)$ have provided a reasonable basis for assessment on numerous projects where empirical performance data are available and are sufficiently comparable to the project under assessment. There is, however, no theoretical foundation to these methods that allows adjustment to cover new or atypical situations. Methods $(a)$ or $(b)$ are commonly used for routine analyses, as simple spreadsheets can be developed which allow the rapid assessment of the required face pressure at numerous sections along the tunnel alignment. It is now common to provide values for target pressures at intervals of $10 \mathrm{~m}$ to $50 \mathrm{~m}$ along a tunnel, requiring 20 to 100 sets of calculations per kilometre of tunnel, and spreadsheet calculations provide a simple means of rapidly and transparently providing the necessary values.

Numerical analyses (method $(c)$ ) are commonly used to assess ground responses in critical locations, as discussed in the report by Golder (2009) and many case histories and research papers. The detailed, non-linear $3 \mathrm{D}$ analysis needed to relate support pressure to surface volume loss requires specialised numerical modelling expertise. Commonly, 2D and $3 \mathrm{D}$ numerical modelling as used in practice does not relate the support pressure and the surface volume loss, but relies on simple assumptions related to ground losses at the face and convergence around the tunnelling systems. This is no different to assuming a value for volume loss and does not provide the necessary linkage between the pressures applied at tunnel level and the resulting volume loss.

The discussers have used published data from the centrifuge testing of model tunnels in clay to calculate face pressures required to limit ground displacements on many tunnelling projects, using simple spreadsheets. Subsequent field monitoring data have shown the robustness of this approach. It would be of potential benefit if charts could be developed relating the tunnel and surface volume loss to the load factor (LF) for tunnels in sand, similar to those available for tunnels in clay (Mair et al., 1981). The necessary support pressure can then be calculated from the load factor that is required to meet the specified limit for volume loss. Such charts could be used either as a basis for the face pressure calculations or the independent checking of calculations.

The discussers have experienced projects with up to seven different parties reviewing the face pressure calculations for PTBM tunnelling, with target pressures based largely on subjective opinion, limited case histories and widely disparate methodologies. The availability of data from parametric centrifuge testing could provide a simple basis for understanding the critical mechanisms involved and resolving differences.

In addition to the authors' work, there have been many papers published summarising the results of centrifuge and $1 \mathrm{~g}$ model testing of tunnels in sand, generally in dense and dry sand (e.g. O'Reilly \& New, 1982; Chambon \& Corte, 1994; Lee et al., 2004; Marshall et al., 2012; Chen et al., 2013; Giardina et al., 2015; Soranzo et al., 2015). These experiments can be separated into two categories: those that examine plane-strain convergence around the tunnel perimeter and those that examine the $3 \mathrm{D}$ effects of ground entry only into the tunnel face. Neither of these types of experiments exactly corresponds to the conditions around a moving PTBM, where loss at the face is supplemented by convergence over the limited distance along the PTBM and during tail void grouting. The limited distance over which convergence can occur over a PTBM means that the behaviour in this area will be dominated by $3 \mathrm{D}$ arching effects, including longitudinal arching between the ground at the front and tail of the PTBM, as illustrated by Wan et al. (2019), which will not be captured in the plane-strain experiments.

Data from plane-strain experiments are summarised in Fig. 16, while tests for deformation at the face only are summarised in Fig. 17. The published data have been evaluated to plot tunnel volume loss against load factor. The load factor (e.g. Atkinson and Potts, 1977; 


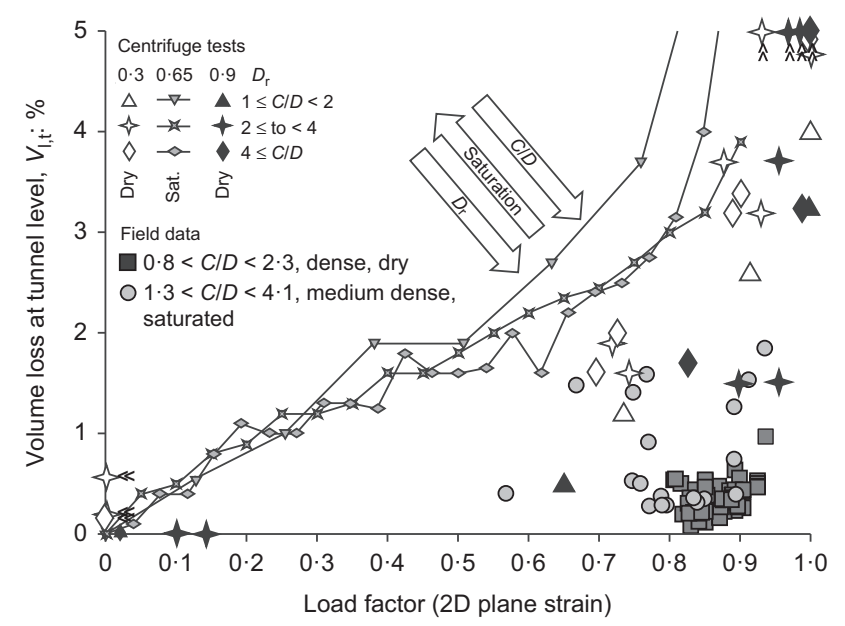

Fig. 16. Summary of centrifuge experiment data for plane-strain (2D) conditions. Centrifuge data for dry conditions at $D_{\mathrm{r}}=0.3$ and 0.9 from Franza et al. (2019), saturated conditions from Lee et al. (2004). Field data taken from Hsiung \& Lu (2008) and Antiga \& Chiorboli (2009)

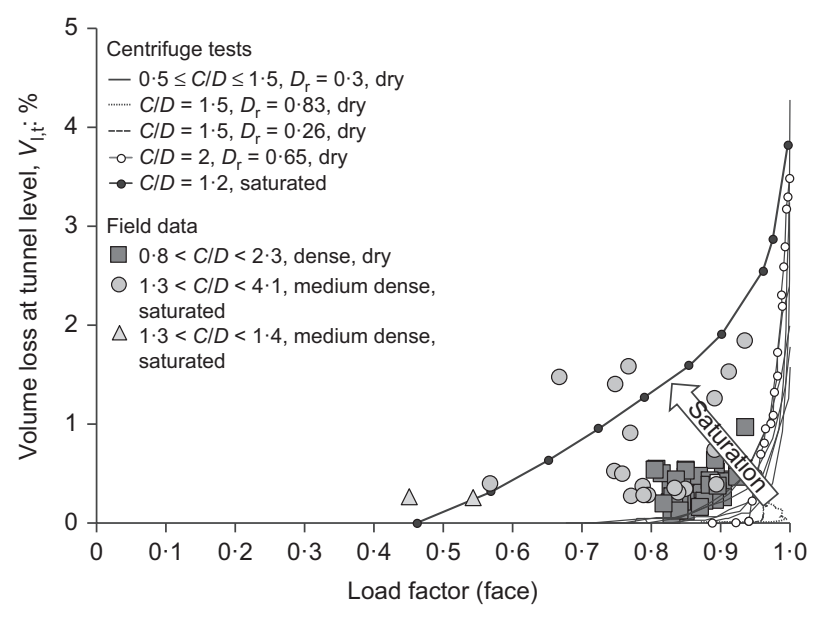

Fig. 17. Summary of centrifuge experiment data for face support conditions. Centrifuge data for dry conditions at $D_{\mathrm{r}}=0 \cdot 3$ and 0.9 from Franza et al. (2019), saturated conditions from Plekkenpol et al. (2006). Field data taken from Orfila et al. (2007), Mignini et al. (2008), Hsiung \& Lu (2008), Antiga \& Chiorboli (2009) and Gens et al. (2012)

O'Reilly, 1988) is a convenient and simple basis of comparison, and is defined as

$$
\frac{\sigma_{\mathrm{vo}}-P_{\mathrm{t}}}{\sigma_{\mathrm{vo}}-P_{\mathrm{c}}}
$$

where $\sigma_{\mathrm{vo}}$ is the total overburden pressure; $P_{\mathrm{t}}$ is the pressure applied within the tunnel to the ground (face or tunnel circumference); and $P_{\mathrm{c}}$ is the tunnel pressure at failure. In terms of effective stress, equation (6) can be written as

$$
\frac{\sigma_{\mathrm{v}}^{\prime}-\Delta P_{\mathrm{t}}}{\sigma_{\mathrm{v}}^{\prime}-\Delta P_{\mathrm{c}}}
$$

where $\Delta P_{\mathrm{t}}$ is the overpressure applied in the tunnel, the overpressure being the pressure in excess of the initial water pressure (Tsang et al., 2012), and $\Delta P_{\mathrm{c}}$ is the overpressure at failure.

The results are identical whether expressed in terms of total or effective stress.

Not all centrifuge tests on model tunnels in sand that have been reviewed were taken to failure. From the published data, the pressure at collapse, $P_{\mathrm{c}}$, was taken as the minimum asymptotic (or nearly so) pressure at which the volume losses represent failure conditions (i.e. $V_{1, t}$ typically $>5 \%$ ). In both Figs 16 and 17, the illustrated volume losses are those at tunnel level.

Although not directly applicable to PTBM tunnelling, Fig. 16 can be used to establish some general relationships

(a) saturating the ground significantly worsens the $V_{1, \mathrm{t}}$ performance, with a higher volume loss for a given value of load factor, compared with dry ground

(b) as $C / D$ increases, the volume loss reduces for a given value of load factor

(c) as the relative density increases, the volume loss reduces for a given value of load factor.

A summary of centrifuge data relating pressure and movement at the face is presented in Fig. 17. Compared with the number of plane-strain tests, there are few published tests that relate the support pressure to the movement at the face of the tunnel, and Fig. 17 includes data from $1 \mathrm{~g}$ as well as centrifuge tests. There are also very few published tests under saturated conditions, rather than in dry sand; a particular exception is a study by Plekkenpol et al. (2006). Most of the testing is for stationary model tunnels in dry sand, while the majority of actual tunnels in sand will be below groundwater levels. As demonstrated in Figs 16 and 17, what evidence there is shows a significant difference between the ground behaviour under dry and saturated conditions. What can be seen in Fig. 17 is the very abrupt change from generally small values of minimal volume loss up to a load factor of $0 \cdot 8$ to $0 \cdot 9$, to the rapid increase in volume loss as the face approaches failure in dry sand. This almost 'brittle' ground behaviour of tunnels in dry sand can be contrasted with the much more ductile behaviour of clays seen in the data presented by Mair et al. (1981) and Macklin (1999).

It is likely that the relationship between tunnel volume loss and load factor for PTBM tunnelling will lie somewhere between the values for movement at the face only (Fig. 17) and the plane-strain tests summarised in Fig. 16.

As well as data from $1 \boldsymbol{g}$ and centrifuge tests, field data published by Antiga \& Chiorboli (2009), Hsiung \& Lu (2008), Orfila et al. (2007) and Gens et al. (2012) are included in Figs 16 and 17 where information was available to discern the appropriate parameters. Field data are shown in both Figs 16 and 17, as separation of face losses and convergence was not fully possible based on the available information.

As a comparison to the dry sand model data, data are shown for a tunnel in Milan completed above the groundwater in dense, granular soils using an earth pressure balance (EPB) TBM (Antiga \& Chiorboli, 2009). Cover-to-diameter ratios ranged from about $0 \cdot 8$ to $2 \cdot 3$. In this case, Antiga \& Chiorboli (2009) present face pressures (EPB chamber pressures) against surface settlement performance, approximately converted to surface volume losses by the discussers for the purpose of this discussion. Almost all data are for surface volume loss of less than $1 \%$, so, based on the authors' work, the volume losses at surface level would be approximately equal to those at tunnel level. As a result, the field data can be compared with the data for tunnel volume loss obtained from the centrifuge testing. Support pressures at collapse were assumed to be consistent with those calculated using the approach of Anagnostou \& Kovari (1996), for the purposes of this discussion. Since an EPB TBM was utilised, there would have been little to no support pressure within the cut-to-shield gap and closure of the shield-to-lining gap would have been controlled by the effectiveness of the tail void grouting. Antiga \& Chiorboli (2009) indicated that approximately $25 \%$ of the volume loss (or settlement) 
occurred at or ahead of the TBM face, with the remainder due to closure of the gap around the TBM and lining, neglecting, however, the time delay associated with vertical propagation of the ground losses in relatively dry ground.

Field data from tunnelling in saturated medium-dense silty sand, below the groundwater table, in Kaohsiung, are also illustrated in Figs 16 and 17 (Hsiung \& Lu, 2008). An EPB TBM was used for this project. Hsiung \& Lu (2008) reported that annular grout volumes ranged between 100 and $150 \%$, indicating that little gap closure occurred and most of the ground losses were associated with face pressure control. Some data from the paper by Hsiung \& Lu (2008) are above $1 \%$ surface volume loss, and are not consistent with the general trend seen in the other points. It is possible that these results show a greater than normal contribution from ground losses due to TBM steering and/or less than ideal tail void grouting. In assessing the data in the paper by Hsiung \& Lu (2008), the methods of Anagnostou \& Kovari (1996) were used for the purposes of defining the pressure at (face) collapse and it was assumed that volume losses at tunnel level were approximately equal to volume losses at the ground surface. Limited data associated with EPB tunnelling below groundwater levels in Barcelona's sandy soils were discerned from work by Orfila et al. (2007), Mignini et al. (2008) and Gens et al. (2012) and are also shown in Fig. 17.

Comparing the field data with the centrifuge test data

(a) the field data show that the effect of saturation is to increase the volume loss for a given value of load factor, compared with dry sand, consistent with the interpretation of the model test results

(b) field data are typically between the 'face only' results and the $2 \mathrm{D}$ results, but closer to the 'face only' results.

The discussers' experience is consistent with the general trends shown in Fig. 17. Provided that an adequate face pressure is applied, and there is effective tail void grouting, the measured values of surface volume loss over tunnels in sand are typically very low. However, if the face pressure falls below an appropriate value, even for a very short time period, the result will be rapidly increasing settlement and/or a sinkhole, depending on the magnitude of the drop in the pressure. For tunnels in saturated sand, the data suggest that maintaining the face pressure so that the load factor is less than about $0 \cdot 8$ is essential for controlling volume losses at tunnel level to less than $1 \%$, under the assumption that gap grouting is fully controlled.

The consequences of not applying correct operating pressures during PTBM tunnelling are potentially severe: excessive settlement, damage to buildings and utilities, and/or a sinkhole. Occasional sinkholes, areas of large local settlement or loss of ground were recorded over many of the EPB driven tunnels for the North East Line in Singapore (Shirlaw et al., 2003), and in many cases were due to an inadequate face pressure being applied. The discussers have observed similar cases of sinkholes over PTBM (both slurry micro-tunnelling and large-diameter tunnelling) elsewhere in Asia and North America. There are many reasons why an inadequate face pressure might be applied, including, based on the discussers' experience

(a) no calculations or estimates of the required face pressures were completed prior to tunnelling

(b) errors were made in the calculations for target face pressures

(c) target face pressure calculations were based on an incorrect geological or hydrogeological model; often far more effort is devoted to assessing the relevant geotechnical parameters for the soil than is devoted to defining the water pressure, which is typically the dominant term in the equations

(d) available geological and hydrogeological data were dismissed in favour of subjectively chosen values

(e) failing to communicate target face pressures to the TBM operator

$(f)$ failure to monitor and adjust face pressures to keep within the required range

(g) failure to achieve the target face pressure by, for example, incorrect conditioning, imbalance between screw conveyor rotation, discharge rates and advance rates in EPB tunnelling or inappropriate slurry properties for slurry TBMs

(h) mechanical failure of the TBM, such as excessive wear of the screw conveyor, or the failure of the main bearing seals or pressure bulkhead

(i) sudden loss of face pressure, due to encountering a well, deep instrumentation or other open path to the ground surface, or flow back into the shaft on launching the TBM

(j) operational personnel being unconcerned about short-term reductions in the face pressure

(k) using an arbitrary basis to develop the allowable range within which face pressures may vary, without assessing the effect on volume loss of tunnelling at the lower end of the range.

Developing the appropriate target values for face, slurry and grouting pressures is an essential step in successful PTBM tunnelling. For the planning, design and construction of tunnels in sand, charts that relate load factor to volume loss, such as have been available for clays for over 30 years, could potentially provide a valuable basis for assessing target values for face and grout pressures. Establishing the relationship between pressure and volume loss is also a tool for focusing risk management on the processes that control ground movements at the tunnel level. Recognising that defining the support pressure at collapse before construction is yet subject to a wide range of opinion (e.g. O'Reilly \& New, 1982; Vermeer et al., 2002), further centrifuge testing and metanalysis of existing data should prove beneficial. Tests that are representative of tunnel heading geometry, and for saturated granular soil conditions, would potentially be of great value.

\section{Authors' reply (A. Franza and A. M. Marshall)}

The authors would like to thank Dr Boone and Mr Shirlaw for their insights relating to some of the current challenges facing tunnelling engineers and for their comments on both the usefulness and limitations of the centrifuge test outcomes presented in the paper by Franza et al. (2019). The submitted discussion is a valuable contribution from the perspective of practitioners and supports the need to move towards more sophisticated reduced scale centrifuge testing, something the authors wholeheartedly agree with. The discussion provides an excellent overview of where new developments in research could provide a direct impact on the tunnelling industry.

\section{SOME CONTEXT TO THE RESEARCH}

In the paper by Franza et al. (2019), the use of dry sand and plane-strain conditions was in part related to an impetus to extend the data set from the paper by Marshall et al. (2012) on tunnelling-induced settlements in dense sand to include medium-dense and loose sands, while also discussing ground 
reaction curves, deformation mechanisms and arching. The $\mathrm{PhD}$ research project conducted by A. Franza focused predominately on tunnel-pile and tunnel-piled building interaction (Franza \& Marshall, 2018, 2019a). For this testing, the use of dry, loose sand allowed for simple, relatively quick and repeatable test preparation. For the interpretation of the outcomes of these tests, the need to better characterise loose sand behaviour under greenfield conditions arose. Furthermore, for the tunnel-pile and tunnel-building interaction experiments, a more simple 2D model was adopted to reduce uncertainties that generally worsen with the complexity of an experimental set-up. Therefore, plane-strain tunnelling in dry sand was considered to be appropriate, which was also consistent with other recent research (Marshall et al., 2010; Farrell et al., 2014; Ritter et al., 2017).

It may also be of interest that the experimental set-up used in the paper by Franza et al. (2019), originally developed by Zhou et al. (2014), was designed to perform tests on saturated soils; future works will deal specifically with aspects highlighted by the discussion relating to the effect of water. In addition, the authors agree with the discussion that centrifuge investigation of 3D tunnelling would be of great interest and practical use; these will also have significant benefit for the study of tunnel-structure interaction problems. It is encouraging that 3D tunnelling systems have been developed for centrifuge testing by using independent ground loss systems (Boonyarak $\& \mathrm{Ng}, 2015)$ and model tunnel-boring machines (Nomoto et al., 1999); more work in this area is certainly warranted.

\section{TUNNEL AND SOIL VOLUME LOSS}

To the authors' knowledge, design values of soil volume losses $V_{1, \mathrm{~s}}$ often range between 0.5 and $2 \%$. The authors referred to $V_{1, \mathrm{t}}=1,2,3$ and $5 \%$ as low, medium, high and extremely high volume losses, respectively, and included the high and very high values to relate to cases where tunnel-boring machines do not achieve the target design soil volume loss, as reported in the discussion. Related to this, Franza \& Marshall (2019b) characterised greenfield ground movements (both vertical and horizontal) from centrifuge test results up to tunnel volume losses $V_{1, \mathrm{t}}=4-5 \%$ with empirical and semi-analytical methods; empirical formulas to estimate (from the considered centrifuge test results) the relationship between $V_{1, \mathrm{~s}}$ and $V_{1, \mathrm{t}}$ as a function of soil relative density $I_{\mathrm{d}}$ and tunnel cover-to-diameter ratio $C / D$ were also provided. Building on what is presented in the discussion, the authors would like to emphasise the importance of predicting both surface and subsurface soil volume losses; while shallow foundations may interact predominately with near-surface movements, buried infrastructure and deep foundations interact with subsurface movements. Surface and subsurface soil volume losses for relative depths of $z / z_{\mathrm{t}}=0,0 \cdot 25,0 \cdot 5$, where $z_{\mathrm{t}}$ is depth to tunnel axis, were reported in both the paper by Franza et al. (2019) (Fig. 9) and that by Franza \& Marshall (2019b).

The discussion highlighted that, for routine design, engineers are interested in the relationship between soil and tunnel volume losses mainly in the range of $V_{1, \mathrm{t}}<2 \%$. To support this, the data from Fig. 9 of the paper by Franza et al. (2019) are reproduced here in Fig. 18 for $V_{1, \mathrm{t}}=0 \cdot 5-2 \%$ and soil relative density $I_{\mathrm{d}}=0.3,0.5$ and 0.9 ; the observed trends agree with the comments within the discussion. Note that information such as that provided within Fig. 18 can be used alongside data on the relationship between $V_{1, t}$ and LF (such as those from the discussion or presented here in Fig. 19) to evaluate soil volume loss at various depths based on a given value of LF.

\section{LOAD FACTOR}

An effective framework for assessing the relationship between tunnel support pressure and surface/sub-surface soil volume loss is certainly needed. The authors are pleased that their research is able to contribute to this goal in some way. Among others, theoretical research recently published by Mo \& Yu (2017) and Vu et al. (2016) has provided the relationship between tunnel support pressure and volume loss for plane-strain and 3D conditions.

The authors would like to add several comments related to the load factor concept provided within the discussion. First consider that the theoretical normalised pressure at the tunnel axis level $\sigma_{\text {norm }}$ is

$$
\sigma_{\mathrm{norm}, 0}=\frac{\sigma_{\mathrm{t}, 0}}{\gamma D}=\frac{\sigma_{\mathrm{v}, 0}}{\gamma D}=\frac{C}{D}+\frac{1}{2}
$$

where $\sigma_{\mathrm{v}, 0}=\gamma(C+D / 2)$ is the vertical stress in the soil at the level of the tunnel axis; $\gamma$ is the soil unit weight; $D$ is the tunnel diameter; $C$ is the ground cover from the surface to the tunnel crown; and the subscripts $\mathrm{t}, \mathrm{v}$, and 0 refer to tunnel, vertical and initial value (prior to volume loss), respectively.

Second, the selection of the tunnel pressure at failure/ collapse $\sigma_{\mathrm{c}}$ (labelled $P_{\mathrm{c}}$ in the discussion) should be discussed. To calculate LF from the data in the paper by Franza et al. (2019), the discussion assumed $\sigma_{\mathrm{c}}$ as the tunnel internal pressure $\sigma_{\mathrm{t}}$ at a tunnel volume loss $V_{1, \mathrm{t}}=5-8 \%$. This value is likely not a true reflection of the collapse pressure from the experiments. For $C / D=2 \cdot 4$ and $I_{\mathrm{d}}=0 \cdot 9$, Marshall (2009) provided data which indicated that collapse was likely reached for $V_{1, \mathrm{t}}=15-20 \%$ with experimental values of

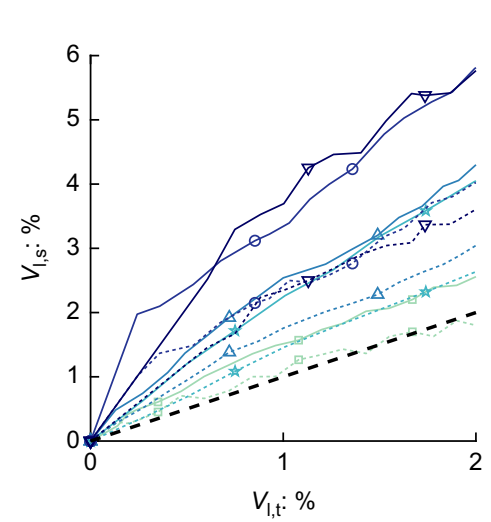

(a)

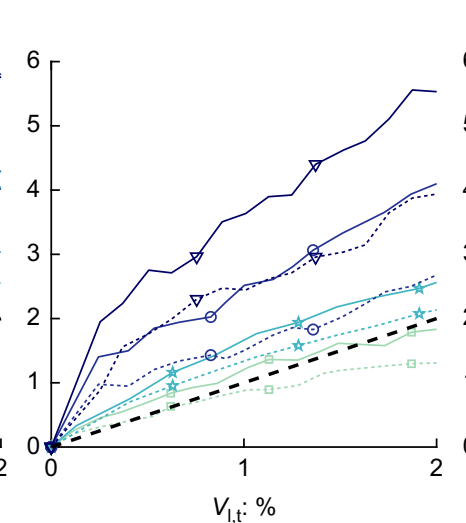

(b)

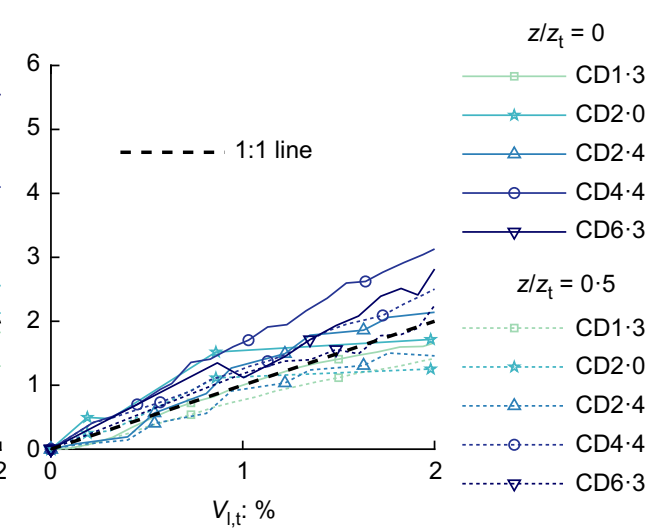

(c)

Fig. 18. $V_{1, \mathrm{~s}}$ plotted against $V_{\mathrm{l}, \mathrm{t}}$ up to $2 \%$ for $z z_{\mathrm{t}}=0$ and $z z_{t}=0 \cdot 5$ : (a) $I_{\mathrm{d}}=0 \cdot 3$; (b) $I_{\mathrm{d}}=0 \cdot 5$; (c) $I_{\mathrm{d}}=0.9$ 


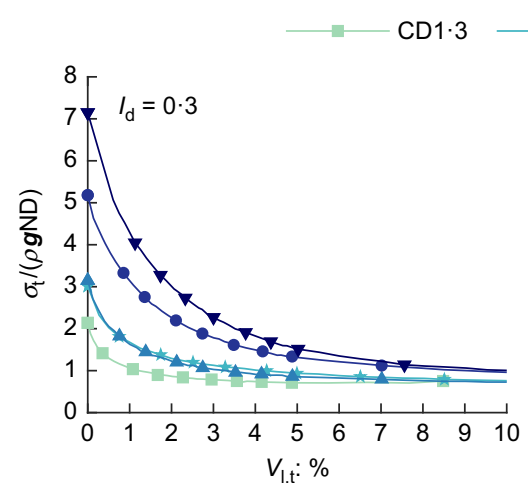

(a)

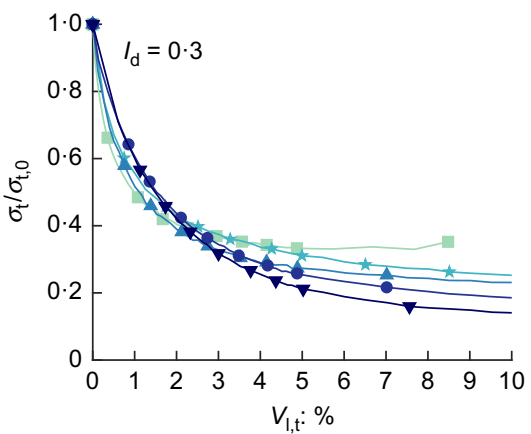

(d)

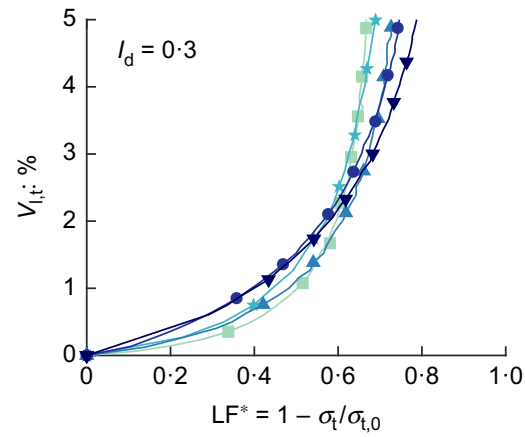

(g)

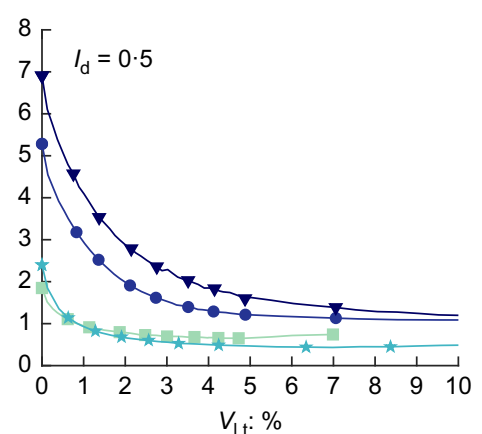

(b)

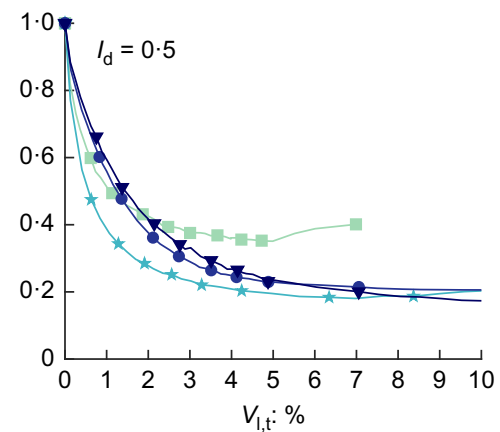

(e)

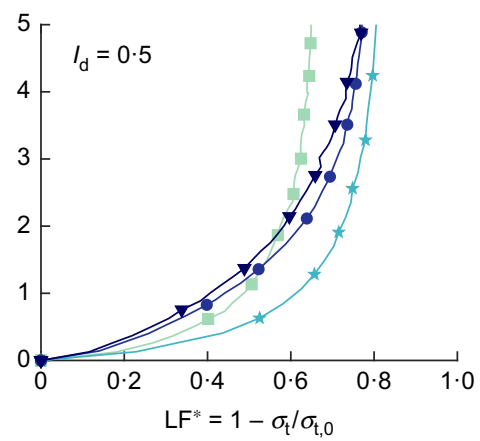

(h)

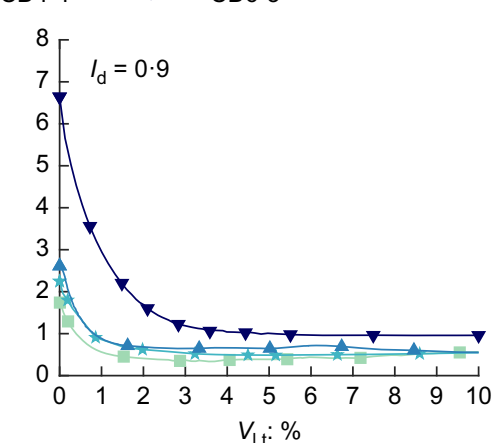

(c)

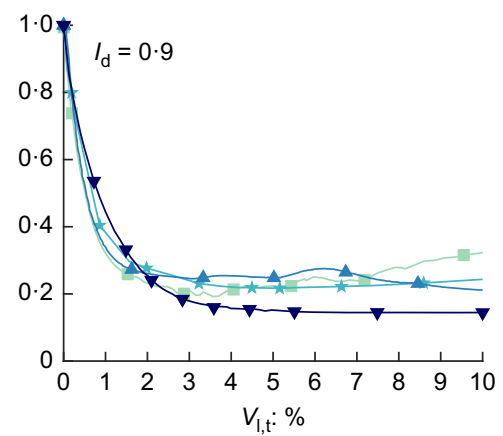

(f)

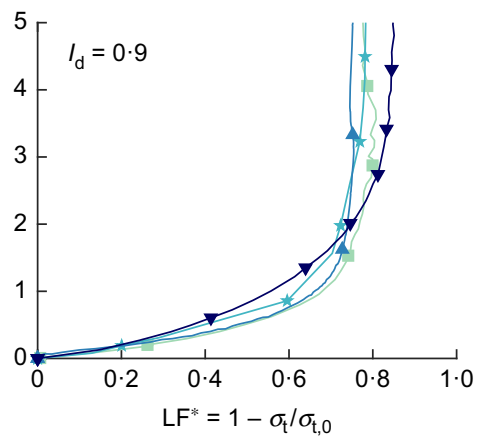

(i)

Fig. 19. (a), (b), (c) Normalised tunnel pressure with tunnel volume loss; (d), (e), (f) relative tunnel pressure with tunnel volume loss; and (g), (h), (i) tunnel volume loss against alternative form of load factor $\mathbf{L F}^{*}$

normalised collapse pressure $\sigma_{\text {norm,c }}=\sigma_{\mathrm{c}} /(\gamma D)$ within the range $0 \cdot 18-0 \cdot 08$. This collapse value is notably lower than the initial $\sigma_{\text {norm, } 0}$ based on equation (8) and smaller than the tunnel support pressure $\sigma_{\text {norm }}$ for $V_{1, \mathrm{t}}=5-8 \%$ used within the discussion. The impact of this feature on the narrative and figures within the discussion is secondary; the conclusions drawn from the interrogation of the Franza et al. (2019) data within the discussion are entirely appropriate.

As discussed above, $\sigma_{\text {norm,c }}$ is much lower than $\sigma_{\text {norm }, 0}$. Considering this, the authors would like to suggest a first (non-conservative) estimate of LF as LF*, which does not require the tunnel collapse pressure, $\sigma_{\mathrm{c}}$

$$
\begin{aligned}
\mathrm{LF}^{*} & =\frac{\sigma_{\mathrm{v}, 0}-\sigma_{\mathrm{t}}}{\sigma_{\mathrm{v}, 0}}=\frac{\sigma_{\mathrm{t}, 0}-\sigma_{\mathrm{t}}}{\sigma_{\mathrm{t}, 0}}=1-\frac{\sigma_{\mathrm{t}}}{\sigma_{\mathrm{t}, 0}} ; \\
\mathrm{LF} & =\frac{\sigma_{\mathrm{v}, 0}-\sigma_{\mathrm{t}}}{\sigma_{\mathrm{v}, 0}-\sigma_{\mathrm{c}}}
\end{aligned}
$$

By definition

$$
\frac{\mathrm{LF}^{*}}{\mathrm{LF}}=\frac{\sigma_{\mathrm{v}, 0}-\sigma_{\mathrm{c}}}{\sigma_{\mathrm{v}, 0}}=1-\frac{\sigma_{\mathrm{c}}}{\sigma_{\mathrm{v}, 0}}=1-\frac{\sigma_{\text {norm }, \mathrm{c}}}{\sigma_{\text {norm }, 0}}<1
$$

For instance, for the test with $C / D=2 \cdot 4$ in dense sand $\left(I_{\mathrm{d}}=0.9\right), \quad \sigma_{\text {norm }, 0}=2.9 \quad$ and $\quad \sigma_{\text {norm }, \mathrm{c}}=0 \cdot 18$ while $\mathrm{LF}^{*}=0.94 \mathrm{LF}$. Thus, $\mathrm{LF}^{*}$ provides a somewhat nonconservative estimate of LF with an error of $6 \%$. As mentioned in the discussion, engineers are interested in prescribing a rational limit for the load factor and $\mathrm{LF}^{*}$ may provide a useful reference for this in cases where there is uncertainty regarding the collapse tunnel pressure. It is worth noting that Franza et al. (2019) reported $\sigma_{t} / \sigma_{t, 0}$ against tunnel volume loss, which is equivalent to the relationship between $\mathrm{LF}^{*}$ and volume loss (refer to equation (9)).

Third, to complement the discussion, the authors have provided here in Fig. 19 the normalised ground reaction curves of the entire available data set; results for medium dense sand $\left(I_{\mathrm{d}}=0 \cdot 5\right)$ were not included in the paper by Franza et al. (2019) due to space restrictions. Figs 19(a)-19(f) show the normalised pressure $\sigma_{\text {norm }}=\sigma_{\mathrm{t}} /(\rho \boldsymbol{g} N D)$ and the relative tunnel pressure $\sigma_{t} / \sigma_{t, 0}$, respectively, against tunnel volume loss. Considering that most of the greenfield tunnelling experiments performed were not taken to a state of full collapse (hence there is uncertainty regarding the value of $\sigma_{\mathrm{c}}$ ), the values of $\mathrm{LF}^{*}$ are used in Figs 19(g)-19(i) rather than LF. 
As mentioned in the discussion, a rather brittle response is obtained for the dense sands, in which the $V_{1, \mathrm{t}}-\mathrm{LF}^{*}$ curves show a vertical asymptotic trend after a certain critical $\mathrm{LF}^{*}$. A brittle tunnel volume loss plotted against $\mathrm{LF}^{*}$ trend is also observed for the shallow tunnels $C / D=1 \cdot 3-2 \cdot 0$ with medium dense sand $\left(I_{\mathrm{D}}=0 \cdot 5\right)$. On the other hand, because of the hardening behaviour of loose sands, the increase of $V_{1, \mathrm{t}}$ with $\mathrm{LF}^{*}$ is more gradual in the loose sand tests. In all cases, the centrifuge data illustrate that increments of $V_{1, \mathrm{t}}$ above $3 \%$ are associated with minimal increases in $\mathrm{LF}^{*}$, confirming the risk related to unexpected large tunnel volume losses above a critical value of load factor.

Fourth, the authors would like to point out that, for model tunnels pressurised with water (as for the paper by Franza et al. (2019)), there is a gradient of internal tunnel pressure across the depth of the tunnel due to the self-weight of the water. As discussed by Farrell (2010), particularly for shallow tunnels, the minimum support pressure at the tunnel crown may lead to a localised collapse in the area around the crown. The impact of this issue on the conclusions provided by the discussion is likely to be insignificant; however, it is mentioned here for the sake of other researchers who are considering centrifuge modelling for the purpose of developing load factor LF design charts.

Finally, it may be of interest that recent research has indicated that the relationship between $V_{1, \mathrm{t}}$ and $V_{1, \mathrm{~s}}$ and, consequently, that between $V_{1, t}$ and LF, is not significantly affected by the presence of a building when compared to the influence of soil density (Ritter et al., 2017).

\section{CONCLUSIONS}

Despite their limitations, plane-strain tests of greenfield tunnelling and tunnel-structure interactions provide a reliable and relatively straightforward way to investigate soil deformation mechanisms. They also provide valuable data for the calibration and validation of analytical and numerical models. However, the authors completely agree with the discussion in that future research could provide significant benefits by investigating more realistic $3 \mathrm{D}$ problems, in terms of improving our understanding of the relationship between load factor and tunnel volume loss, as well as the prediction of tunnel-structure interactions. The authors would like to emphasise the importance of continuous and open discussions between researchers and practitioners, and would again like to thank the discussion contributors for their insightful contribution, which has provided guidance for the direction of the authors' future research initiatives.

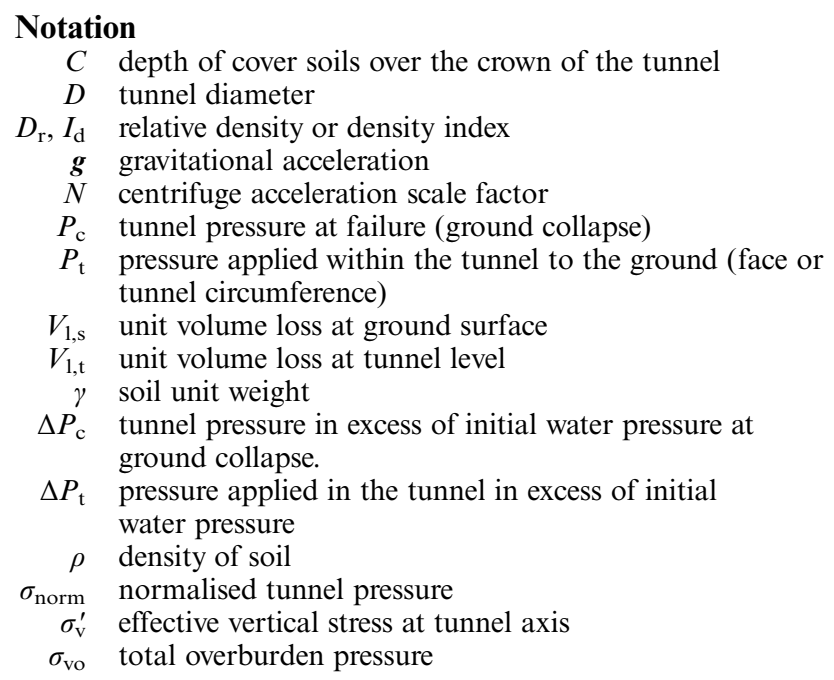

\section{REFERENCES}

Anagnostou, G. \& Kovari, K. (1996). Face stability in slurry and EPB shield tunnelling. Proceedings of the symposium on geotechnical aspects of underground construction in soft ground, London, UK, pp. 379-384.

Antiga, A. \& Chiorboli, M. (2009). Tunnel face stability and settlement control using earth pressure balance shield in cohesionless soil. In Geotechnical aspects of underground construction in soft ground (eds C. W. W. Ng, H. W. Huang and G. B. Liu), pp. 365-371. Boca Raton, FL, USA: CRC Press/Balkema.

Atkinson, J. H. \& Mair, R. J. (1981). Soil mechanics aspects of soft ground tunnelling. Ground Engng 14, No. 5, 20-26.

Atkinson, J. H. \& Potts, D. M. (1977). Subsidence above shallow tunnels in soft ground. J. Geotech. Engng Div., ASCE 103, No. GT4, 307-326.

Boonyarak, T. \& Ng, C. W. W. (2015). Effects of construction sequence and cover depth on crossing-tunnel interaction. Can. Geotech. J. 52, No. 7, 851-867.

CEDD (Civil Engineering and Development Department) (2014). Ground control for EPB TBM tunnelling, GEO Report No. 298. Hong Kong, Hong Kong SAR: Civil Engineering and Development Department, The Government of the Hong Kong Special Administrative Region.

Chambon, P. \& Corte, J.-F. (1994). Shallow tunnels in cohesionless soil: stability of tunnel face. J. Geotech. Engng 120, No. 7, 1148-1165.

Chen, R., Li, J., Kong, L. \& Tang, L. (2013). Experimental study on face instability of shield tunnel in sand. Tunnelling Underground Space Technol. 33, 12-21.

DAUB (Deutcher Ausschuss für Unterirdisches Bausen) (2016). Recommendations for face pressure support calculations for shield tunnelling in soft ground. Cologne, Germany: DAUB, Deutcher Ausschuss für Unterirdisches Bausen, German Tunnelling Committee (ITA-AITES).

Farrell, R. (2010). Tunnelling in sands and the response of buildings. PhD thesis, Cambridge University, Cambridge, UK.

Farrell, R., Mair, R., Sciotti, A. \& Pigorini, A. (2014). Building response to tunnelling. Soils Found. 54, No. 3, 269-279, https://doi.org/10.1016/j.sandf.2014.04.003.

Franza, A. \& Marshall, A. M. (2018). Centrifuge modeling study of the response of piled structures to tunneling. J. Geotech. Geoenviron. Engng 144, No. 2, 04017109, https://doi.org/ 10.1061/(ASCE)GT.1943-5606.0001751.

Franza, A. \& Marshall, A. M. (2019a). Centrifuge and real-time hybrid testing of tunneling beneath piles and piled buildings. J. Geotech. Geoenviron. Engng 145, No. 3, 04018110, https:// doi.org/10.1061/(ASCE)GT.1943-5606.0002003.

Franza, A. \& Marshall, A. M. (2019b). Empirical and semianalytical methods for evaluating tunnelling-induced ground movements in sands. Tunneling Underground Space Technol. 88, 47-62, https://doi.org/10.1016/j.tust.2019.02.016.

Franza, A., Marshall, A. M. \& Zhou, B. (2019). Greenfield tunnelling in sands: the effects of soil density and relative depth. Géotechnique 69, No. 4, 297-307, https://doi.org/ 10.1680/jgeot.17.p.091.

Gens, A., DiMariano, A. \& Yubero, M. T. (2012). EPB tunnelling in deltaic deposits: observations of ground movements. In Geotechnical aspects of underground construction in soft ground (ed. J. G. Viggiani), pp. 987-993. London, UK: Taylor \& Francis.

Giardina, G., DeJong, M. J. \& Mair, R. J. (2015). Interaction between surface structures and tunnelling in sand: centrifuge and computational modelling. Tunnelling Underground Space Technol. 50, 465-478, https://doi.org/10.1016/j.tust.2015.07.016.

Golder Associates (HK) Ltd. (2009). Ground control for slurry TBM tunnelling, GEO Report No. 249. Hong Kong, Hong Kong SAR: Civil Engineering and Development Department, The Government of the Hong Kong Special Administrative Region.

Hsiung, B. B. C. \& Lu, K. L. (2008). A bored tunnel on kaohsiung rapid transit system, contract CR2. J. Geoengng, Taiwan Geotech. Soc. 3, No. 1, 33-40.

Iglesia, G. R., Einstein, H. H. \& Whitman, R. V. (2013). Investigation of soil arching with centrifuge tests. J. Geotech. Geoenviron. Engng, ASCE 140, No. 2, https://doi.org/10.1061/ (ASCE)GT.1943-5606.0000998. 
Kimura, T. \& Mair, R. J. (1981). Centrifugal testing of model tunnels in soft clay. In Proceedings of the 10th international conference on soil mechanics and foundation engineering (ed. Publications Committee of XICSMFE), vol. 1, pp. 319-322. Rotterdam, the Netherlands: Balkema.

Lee, C. J., Chiang, K. H. \& Kuo, C. M. (2004). Ground movement and tunnel stability when tunneling in sandy ground. J. Chin. Inst. Engrs 27, No. 7, 1021-1032.

Macklin, S. R. (1999). The prediction of volume loss due to tunnelling in overconsolidated clay based on heading geometry and stability number. Ground Engng 32, No. 4, 30-33.

Mair, R. J., Gunn, M. J. \& O'Reilly, M. P. (1981). Ground movements around shallow tunnels in soft clay. In Proceedings of the 10th international conference on soil mechanics and foundation engineering (ed. Publications Committee of XICSMFE), vol. 1, pp. 323-328. Rotterdam, the Netherlands: Balkema.

Marshall, A. M. (2009). Tunnelling in sand and its effect on pipelines and piles. $\mathrm{PhD}$ thesis, Cambridge University, Cambridge, UK.

Marshall, A. M., Klar, A. \& Mair, R. J. (2010). Tunneling beneath buried pipes: view of soil strain and its effect on pipeline behavior. J. Geotech. Geoenviron. Engng 136, No. 12, 1664-1672, https://doi.org/10.1061/(ASCE)GT.1943-5606.0000390.

Marshall, A. M., Farrell, R., Klar, A. \& Mair, R. (2012). Tunnels in sands: the effect of size, depth and volume loss on greenfield displacements. Géotechnique 62, No. 5, 385-399, https://doi.org/ 10.1680/geot.10.P.047.

Mignini, A., Orfila, T., Colomer, M. \& Bertagnolio, F. (2008). Surface settlement minimization in soft soil when excavating with an earth pressure balance shield. Barcelona metro line 9 Mas Blau-San Cosme station. Tunnelling working procedure. In Jornada técnica: túneles con EPB. Simulación y control de la tuneladora (eds E. Alonso and M. Arroyo), pp. 149-162. Barcelona, Spain: Universitat Politècnica de Catalunya.

Mo, P. Q. \& Yu, H. S. (2017). Undrained cavity-contraction analysis for prediction of soil behavior around tunnels. Int. J. Geomech. 17, No. 5, 04016121, https://doi.org/10.1061/(ASCE)GM. 1943-5622.0000816.

New, B. M. \& O'Reilly, M. P. (1981). Tunnelling induced ground movements: predicting their magnitude and effects. In Proceedings of the 4th international conference on ground movements and structures (ed. J. D. Geddes), pp. 671-697. London, UK: Pentech Press.

Nomoto, B. T., Imamura, S., Hagiwara, T., Kusakabe, O. \& Fujii, N. (1999). Shield tunnel construction in centrifuge. J. Geotech. Geoenviron. Engng 125, No. 4, 289-300.

O'Reilly, M. P. (1988). Evaluating and predicting ground settlements caused by tunnelling in London Clay. In Tunnelling '88: papers presented at the fifth international symposium (ed. M. J. Jones), pp. 231-241. London, UK: The Institution of Mining and Metallurgy.

O'Reilly, M. P. \& New, B. M. (1982). Settlements above tunnels in the United Kingdom - their magnitude and prediction. In Tunnelling '82: papers presented at the third international symposium (ed. M. J. Jones), pp. 173-181. London, UK: Institution of Mining and Metallurgy.
Orfila, T., Moya, N. \& Della Valle, N. (2007). Optimizing Line 9's EPBM parameters. Tunnels and Tunnelling International April, pp. $40-45$.

Peck, R. B. (1969). Deep excavations and tunnelling in soft ground. In Proceedings of the 7th international conference on soil mechanics and foundation engineering (ed. Papers Subcommittee of VII ICSMFE), pp. 225-290. Mexico City, Mexico: Sociedad Mexicanos de Mecánica de Suelos.

Plekkenpol, J. W., van der Schrier, J. S. \& Hergarden, H. J. (2006). Shield tunnelling in saturated sand - face support pressure and soil deformations. In Tunnelling: a decade of progress, Geo-Delft 1995-2005 (eds A. Bezuijen and H. van Lottum), pp. 133-142. London, UK: Taylor \& Francis.

Ritter, S., Giardina, G., DeJong, M. J. \& Mair, R. J. (2017). Influence of building characteristics on tunnelling-induced ground movements. Géotechnique 67, No. 10, 926-937, https://doi.org/10.1680/jgeot.SIP17.P.138.

Shirlaw, J. N. (2012). Setting operating pressures for TBM tunnelling. Keynote lecture. In Geotechnical aspects of tunnelling for infrastructure development: proceedings of the HKIE Geotechnical Division 32nd annual seminar, pp. 7-28. Hong Kong, China: Hong Kong Institution of Engineers.

Shirlaw, J. N. \& Boone, S. J. (2009). Geotechnical engineering for TBM tunnelling in soft and mixed ground. Geotech. Engng, J. South East Asian Geotech. Soc. 40, No. 2, 107-116.

Shirlaw, J. N., Ong, J. C. W., Rosser, H. B., Tan, C. G., Osborne, N. H. \& Heslop, P. J. E. (2003). Local settlements and sinkholes due to EPB tunnelling. Proc. Instn Civ. Engrs Geotech. Engng 56, No. GE4, 193-211, https://doi.org/10.1680/ geng.2003.156.4.193.

Soranzo, E., Tamagnini, R. \& Wu, W. (2015). Face stability of shallow tunnels in partially saturated soil: centrifuge testing and numerical analysis. Géotechnique 65, No. 6, 454-467, https://doi.org/10.1680/geot.14.P.123.

Tsang, A. C. M., Salisbury, C. D. \& Yeung, S. S. M. (2012) Confinement pressure for face stability of tunnel boring machine (TBM) tunnel excavation under Hong Kong's western district. In Geotechnical aspects of tunnelling for infrastructure development: proceedings of the HKIE Geotechnical Division 32nd annual seminar, pp. 147-158. Hong Kong, China: Hong Kong Institution of Engineers.

Vermeer, P. A., Ruse, N. M. \& Marcher, T. (2002). Tunnel heading stability in drained ground. Felsbau 20, No. 6, 8-18.

$\mathrm{Vu}, \mathrm{M}$. N., Broere, W. \& Bosch, J. (2016). Volume loss in shallow tunnelling. Tunnelling Underground Space Technol. 59, 77-90, https://doi.org/10.1016/j.tust.2016.06.011.

Wan, M. S. P., Standing, J. R., Potts, D. M. \& Burland, J. B. (2019). Pore water pressure and total horizontal stress response to EPBM tunnelling in London Clay. Géotechnique 69, No. 5, 434 457, https://doi.org/10.1680/jgeot.17.p.309.

Zhou, B., Marshall, A. M. \& Yu, H. S. (2014). The effect of relative density on greenfield settlements above tunnels in sands. In Geo-Shanghai 2014: tunneling and underground construction (eds W. Ding and X. Li), GSP 242, pp. 96-105. Reston, VA, USA: American Society of Civil Engineers (ASCE). 